\title{
Disorder-Induced Long-Ranged Correlations in Scalar Active Matter
}

\author{
Sunghan Ro, ${ }^{1}$ Yariv Kafri, ${ }^{1}$ Mehran Kardar, ${ }^{2}$ and Julien Tailleur ${ }^{3}$ \\ ${ }^{1}$ Department of Physics, Technion-Israel Institute of Technology, Haifa, 3200003, Israel. \\ ${ }^{2}$ Department of Physics, Massachusetts Institute of Technology, Cambridge, Massachusetts 02139, USA. \\ ${ }^{3}$ Université de Paris, laboratoire Matière et Systèmes Complexes (MSC), UMR 7057 CNRS, 75205 Paris, France.
}

\begin{abstract}
We study the impact of quenched random potentials and torques on scalar active matter. Microscopic simulations reveal that motility-induced phase separation is replaced in two-dimensions by an asymptotically homogeneous phase with anomalous long-ranged correlations and non-vanishing steady-state currents. Using a combination of phenomenological models and a field-theoretical treatment, we show the existence of a lower-critical dimension, $d_{c}=4$, below which phase separation is only observed for systems smaller than an Imry-Ma length-scale. We identify a weak-disorder regime in which the structure factor scales as $S(q) \sim 1 / q^{2}$ which accounts for our numerics. In $d=2$ we predict that, at larger scales, the behaviour should cross over to a strong-disorder regime. In $d>2$, these two regimes exist separately, depending on the strength of the potential.
\end{abstract}

The influence of disorder on active systems has attracted a lot of interest recently [1 14]. In particular, long-range order has shown a surprising stability against the introduction of quenched disorder 15 21]. For systems belonging to the Vicsek universality class, where the order parameter has a continuous symmetry, the lower critical dimension was shown to be $d_{c}=2$ : long-ranged polar order is observed in $d=3$ and quasi-long-ranged order in $d=2$ [16 18]. This makes such active systems more robust to disorder than equilibrium ones with a continuous symmetry, for which $d_{c}=4$ [22 28].

While a lot of effort has been devoted to polar aligning active matter, comparatively less is known on the influence of disorder on the collective properties of scalar active matter, when the sole hydrodynamic mode is the density field. There, the combination of self-propulsion and kinetic hindrance leads to motility-induced phase separation (MIPS), even in the absence of attractive interactions, in dimensions $d \geq 2$ 29 46]. Despite important differences, MIPS shares many features of an equilibrium liquid-gas phase separation. The latter is stable to disorder above a lower-critical dimension $d_{c}=2$, and it is natural to ask whether the same holds for MIPS.

In this Letter, we address this question by studying model systems of scalar active matter in the presence of quenched random potentials and torques using a combination of analytical and numerical approaches. The relevance of our results to experimental systems is discussed in the conclusion. We show that MIPS is destroyed for $d \leq d_{c}$ with $d_{c}=4$ : The system only looks phase separated below an Imry-Ma length scale. Instead, disorder leads to asymptotically homogeneous systems with persistent steady-state currents. For $d>2$, the system is either in a weak-disorder regime or in a strongdisorder one depending on the strength of the random potential. In the weak-disorder regime, the system is shown to exhibit self-similar correlations with a structure factor decaying as a power law, $S(q) \sim q^{-2}$, at small wave numbers $q$. This behavior is very different from that of an equilibrium scalar system, where correlations are short-ranged with a structure factor behaving as a Lorentzian squared [28, 47]. In $d=2$, we instead predict a crossover between weak- and strong-disorder regimes at a length scale that we identify. Numerically we only observe the weak-disorder regime, in which we measure a pair-correlation function that decays logarithmically, in agreement with our analytical predictions. Interestingly our results show that, contrary to what was reported for the transition to collective motion [16, 17], scalar active systems are more fragile to disorder than passive ones. Our results are presented for random potentials but naturally extend to random torques, as shown in SI [48].

Numerical simulations. We start by presenting results from numerical simulations of $N$ run-and-tumble particles (RTPs) with excluded volume interactions on a twodimensional lattice [42 44, 49, 50] of size $L \times L$ and pe-
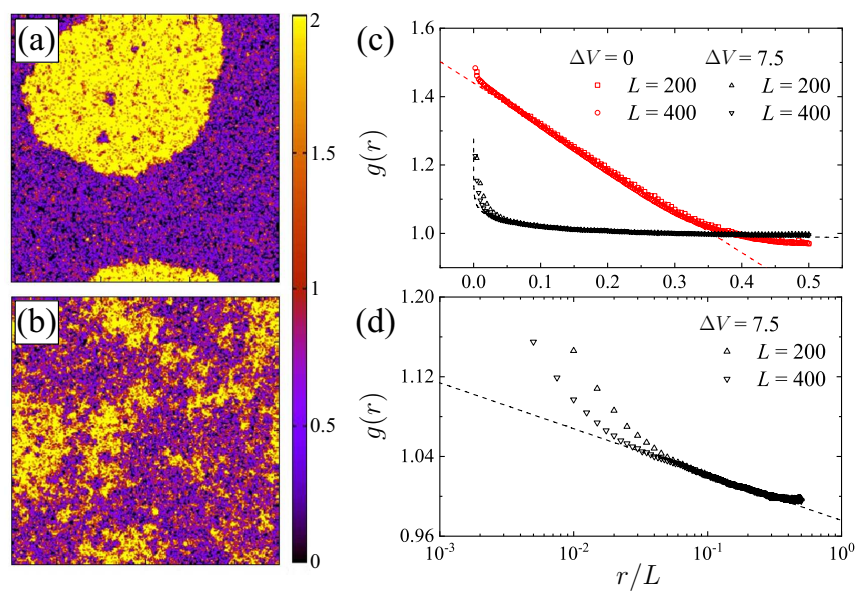

FIG. 1. Snapshots of simulations without (a) and with (b) disorder. Color encodes density, obtained by averaging occupancies over 4 neighbouring sites. (c) The pair correlation functions are shown in linear scale with (black) and without (red) disorder. (d) Pair correlation with disorder using log-linear scale. The dashed lines correspond to linear (red) and logarithmic (black) decays. Parameters: $L=300(\mathrm{a}-\mathrm{b})$, $\Delta V=7.5(\mathrm{~b}), v=13, \alpha=1, n_{M}=2, \rho_{0} \equiv N / L^{2}=1$. 
riodic boundary conditions. Each particle has an orientation $\hat{e}_{\theta}=(\cos \theta, \sin \theta)$ with $\theta \in[0,2 \pi)$, and reorients to a new random direction with rate $\alpha$. In the absence of disorder, activity is accounted for by hops from the position $\vec{i}$ of a particle to any neighboring site $\vec{j}=\vec{i}+\hat{u}$ with rate $W_{\vec{i}, \vec{j}}=\max \left[v \hat{u} \cdot \hat{e}_{\theta}, 0\right]$, where $v$ controls the propulsion speed. Interactions between the particles are accounted for by modifying the hoping rates according to $W_{\vec{i}, \vec{j}}^{\text {int }}=W_{\vec{i}, \vec{j}}\left(1-n_{\vec{j}} / n_{M}\right)$ with $n_{\vec{j}}$ the number of particles at $\vec{j}$ and $n_{M}$ the maximal occupancy. For large enough $v / \alpha$ and densities, as shown in Fig. 1(a), the system displays MIPS [42]. The quenched disorder is modeled using $W_{\vec{i}, \vec{j}}=\max \left[v \hat{u} \cdot \hat{e}_{\theta}-\left(V_{\vec{j}}-V_{\vec{i}}\right), 0\right]$ with $V_{\vec{i}}$ a random potential drawn from a bounded uniform distribution, $V_{\vec{i}} \in[-\Delta V, \Delta V]$. Here, the lattice spacing and the mobility are set to one.

Surprisingly, Fig. 1(b) suggests that the phase separation is washed out by the random potential. The resulting disordered phase displays, however, a non-trivial structure, suggestive of interesting correlations. We quantify the latter using the pair correlation function $g(r)=\overline{\frac{1}{L^{2}} \sum_{j}\left\langle n_{\vec{j}} n_{\vec{j}+\vec{r}}\right\rangle}$ where $r \equiv|\vec{r}|$, the brackets represent a steady-state average, and the overline an average over disorder realisations. In the absence of disorder, phase separation translates into a linear decay of $g(r)$, as shown in Fig. 1(c). On the contrary, in the presence of disorder, the correlations are found to decay logarithmically, $g(r) \sim \log (L / r)$, as shown in Fig. 1(d). This corresponds to a structure factor $S(q) \sim q^{-2}$ for small $q$.

To explain the disappearance of phase separation and the emergence of non-trivial correlations, we first introduce a phenomenological model which captures the latter in a dilute system. This then suggests a field-theoretic perspective which predicts the existence of weak- and strong-disorder regimes. It first allows us to characterize the disorder-induced persistent currents that flow in the system and then to come back to the arrest of MIPS. Using the field theory, we identify the lower critical dimension as $d_{c}=4$ and estimate the Imry-Ma length scale above which phase separation is arrested in $d<4$.

Phenomenological model for a dilute system. Random potential and torques impact many aspects of the singleparticle dynamics. As we show, all the emerging phenomenology reported here can be traced back to a single aspect: the emergence of ratchet currents. When a localized asymmetric potential centered around $\mathbf{r}_{0}$ is placed in an active fluid of non-interacting RTPs, the stationary density field $\langle\rho(\mathbf{r})\rangle$ in the far field of the potential is 51 .

$$
\langle\rho(\mathbf{r})\rangle=\rho_{0}+\frac{\beta_{\mathrm{eff}}}{S_{d}} \frac{\left(\mathbf{r}-\mathbf{r}_{0}\right) \cdot \mathbf{p}}{\left|\mathbf{r}-\mathbf{r}_{0}\right|^{d}}+\mathcal{O}\left(\left|\mathbf{r}-\mathbf{r}_{0}\right|^{-d}\right) .
$$

Here, $S_{d}=2 \pi^{d / 2} / \Gamma(d / 2), \rho_{0}$ is the density of the active fluid, $\beta_{\text {eff }} \equiv 2 \alpha / v^{2}$, and the mobility of the particles is set to one. The vector $\mathbf{p}$ is given by the average force exerted by the potential on the active fluid and is thus

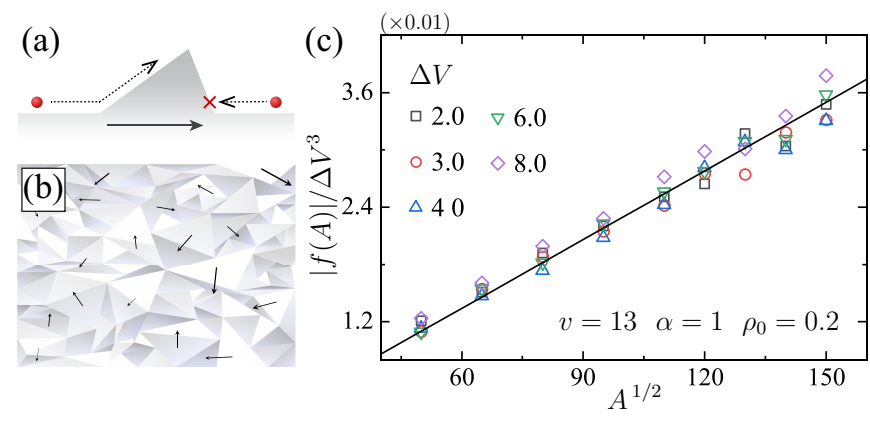

FIG. 2. (a) In $d=1$, asymmetric potentials leads to a nonzero average force on the particles, indicated by a bold arrow. This is accompanied by a non-vanishing ratchet current. (b) In $d=2$, a random potential leads to a steady-state field of random forces exerted on the particles. (c) Measurement of the force, $f(A)$, exerted on the active particles inside a region of area $A$ in the presence of a random potential. The amplitude of the force is quantified by $|f(A)| \equiv\left(\overline{f^{2}(A)}\right)^{1 / 2}$, where $f(A)$ is obtained by time-averaging $\sum_{\vec{i} \in A} n_{\vec{i}}\left(V_{\vec{i}+\vec{e}}-\right.$ $\left.V_{\vec{i}-\vec{e}}\right) / 2$ with $\vec{e}$ an arbitrary unit vector.)

proportional to the overall density. In the presence of torques, Eq. (11) still holds but with a renormalized $\mathbf{p}$ [48]. Given the analogy between Eq. (11) and electrostatics, we follow Ref. 51] and refer to the force $\mathbf{p}$ as a dipole.

With Eq. (1) in mind, we consider a phenomenological model in which the bounded random potential is modelled as a superposition of random independent dipoles. Each dipole exerts a force on the active particles in a direction dictated by the local potential, as sketched in Figs. 2(a) and (b). To test this random dipole picture numerically, we measure in Fig. 2(c) the force $f(A)$ exerted along an arbitrary direction by the random potential on the particles inside an area $A$. Consistent with our phenomenological model, $f(A)$ scales as $\sqrt{A}$. This should be contrasted with equilibrium systems, where $f(A)$ is expected to scale as $A^{1 / 4}$. Indeed, a random bounded potential $V(\mathbf{r})$ leads, in equilibrium, to a force density $\propto \beta^{-1} \rho_{0} \nabla \exp (-\beta V)$. Integrating over an area $A$ solely leads to a boundary contribution proportional to $\int_{\partial A} \exp (-\beta V) \vec{n} d \ell$. Only the fluctuations of $V(\mathbf{r})$ contribute, leading to the $A^{1 / 4}$ scaling. Figure 2(c) thus highlights the non-equilibrium nature of the system: the ratchet effect induced by the random potential leads to an emerging force field with short-range correlations. Finally, the scaling of $F(A)$ as $\Delta V^{3}$ in this dilute regime is consistent with a perturbative result which predicts $|\mathbf{p}| \sim \Delta V^{3}$ as $\Delta V \rightarrow 0$ [51], despite the relatively large values of $\Delta V$ used here.

We now use the phenomenological model to predict the structure factor based on the random dipole picture. The dipole density field $\mathbf{P}(\mathbf{r})$ is randomly drawn from a distribution such that the spatial components of $\mathbf{P}$ satisfy $\overline{P_{i}(\mathbf{r})}=0$ and $\overline{P_{i}(\mathbf{r}) P_{j}\left(\mathbf{r}^{\prime}\right)}=\chi^{2} \delta_{i j} \delta^{d}\left(\mathbf{r}-\mathbf{r}^{\prime}\right)$, notably lacking spatial correlations in $\mathbf{P}(\mathbf{r})$. Denoting $\langle\phi(\mathbf{r})\rangle \equiv$ 
$\langle\rho(\mathbf{r})\rangle-\rho_{0}$, a direct computation, detailed in [48], leads from Eq. (1) to the disorder-averaged structure factor:

$$
S(\mathbf{q}) \equiv \overline{\langle\phi(\mathbf{q}) \phi(-\mathbf{q})\rangle}=\frac{\beta_{\mathrm{eff}}^{2} \chi^{2}}{q^{2}},
$$

with $q \equiv|\mathbf{q}|$. Note that, in the dilute (noninteracting) regime, the computation simplifies thanks to $S(\mathbf{q})=$ $\overline{\langle\phi(\mathbf{q})\rangle\langle\phi(-\mathbf{q})\rangle}$. Including interactions between the particles is possible at the level of Eq. (11) 52], which would only change the prefactor of $q^{-2}$ in Eq. (2). We stress that these predictions, illustrated for scalar active matter, should hold for many active systems, including polar and nematic ones, in the disordered phase. In [48] we indeed report long-range correlations in a dilute system with aligning interactions.

Remarkably, the functional form of $S(\mathbf{q})$ predicted by the phenomenological model agrees well with our numerics even when the particle density is not small, see Fig. 1(d). Building on the successful predictions of the phenomenological model, we now propose a fieldtheoretical description of scalar active matter subject to quenched random potentials.

Field-theoretic treatment. Our results suggest that the overall density is homogeneous at large scales, with small fluctuations, so that the system can be described by a linear field theory. This assumption will be checked in the Section Strong disorder regime using a self-consistency criterion. To model the force field emerging from the ratchet effect due to the bounded random potential $V(\mathbf{r})$, we introduce a quenched random force density $\mathbf{f}(\mathbf{r})$ acting on the active fluid. We consider the dynamics:

$$
\begin{aligned}
\frac{\partial}{\partial t} \phi(\mathbf{r}, t) & =-\nabla \cdot \mathbf{j}(\mathbf{r}, t), \\
\mathbf{j}(\mathbf{r}, t) & =-\nabla \mu[\phi]+\mathbf{f}(\mathbf{r})+\sqrt{2 D} \boldsymbol{\eta}(\mathbf{r}, t),
\end{aligned}
$$

where $\phi(\mathbf{r}, t)$ denotes density fluctuations, $\mathbf{j}(\mathbf{r}, t)$ is the linearized current, and $\boldsymbol{\eta}(\mathbf{r}, t)$ is a unit Gaussian white noise field. The mobility is set to one and the randomforce density satisfies $\overline{f_{i}(\mathbf{r})}=0$ and $\overline{f_{i}(\mathbf{r}) f_{j}\left(\mathbf{r}^{\prime}\right)}=$ $\sigma^{2} \delta_{i j} \delta^{d}\left(\mathbf{r}-\mathbf{r}^{\prime}\right)$. To linear order in $\phi$ we set

$$
\mu[\phi(\mathbf{r}, t)]=u \phi(\mathbf{r}, t)-K \nabla^{2} \phi(\mathbf{r}, t),
$$

with $u, K>0$ to ensure stability. Note that, in the smallfluctuation regime, $\boldsymbol{\eta}$ and $\mathbf{f}$ are independent of $\phi$, while $\sigma$, $D, u$ and $K$ generically depend on the mean density $\rho_{0}$. Much work has been done, in other contexts, on a single particle subject to a random force [53, 54]. Our results complement these classical works at the level of collective modes. The structure factor is then given by [48]:

$$
S(\mathbf{q})=\frac{\sigma^{2}}{q^{2}\left(u+K q^{2}\right)^{2}}+\frac{D}{\left(u+K q^{2}\right)} .
$$

Note that the small $q$ behavior of the structure factor reproduces the scaling $S(q) \propto q^{-2}$ predicted by the phenomenological model and observed in the numerics of Fig.
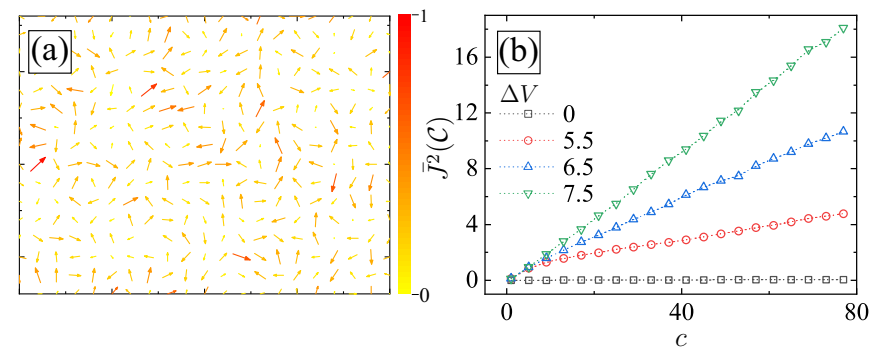

FIG. 3. The current induced by disorder and its statistical properties. (a) Current vector map for a realization of disorder. The color code is the steady-state current normalized by the maximum value measured. (b) The variance of the sum of current $J(\mathcal{C})$ along a contour $\mathcal{C}$ as a function of the its perimeter $c$. Parameters: $v=13, \alpha=1, \Delta V=6.5$ in (a).

1. In fact, comparing Eqs. (2) and (6) shows that $\sigma / u$ is proportional, in the dilute regime, to the inverse effective temperature: $\sigma / u \propto \chi \beta_{\text {eff }}$ [55]. Interestingly, noise and interactions are subleading as $q \rightarrow 0$.

To further understand this result, we use a HelmholtzHodge decomposition of the random force field: $\mathbf{f}(\mathbf{r})=$ $-\nabla U(\mathbf{r})+\boldsymbol{\xi}(\mathbf{r})$. Here $U(\mathbf{r})$ is an effective potential reconstructed from the random force. Its statistical properties, as we show below, are very different from those of the potential $V(\mathbf{r})$ which is short-range correlated. The reconstructed vector field $\boldsymbol{\xi}(\mathbf{r})$ satisfies $\nabla \cdot \boldsymbol{\xi}(\mathbf{r})=0$, so that it impacts the current $\mathbf{j}$ but does not influence the dynamics of the density field. To enforce the delta correlations of $\mathbf{f}(\mathbf{r})$ together with its statistics, we set $\overline{U(\mathbf{q}) U\left(\mathbf{q}^{\prime}\right)}=$ $\sigma^{2} q^{-2} \delta_{\mathbf{q},-\mathbf{q}^{\prime}}^{d}, \overline{\xi_{i}(\mathbf{q}) \xi_{j}\left(\mathbf{q}^{\prime}\right)}=\sigma^{2}\left(\delta_{i j}-q_{i} q_{j}^{\prime} / q^{2}\right) \delta_{\mathbf{q},-\mathbf{q}^{\prime}}^{d}$, and $\overline{U(\mathbf{q}) \boldsymbol{\xi}\left(\mathbf{q}^{\prime}\right)}=0$ so that $U$ and $\xi$ are intimately related. Inserting the decomposition into Eqs. (3) and (44) shows that the density fluctuations of active particles in the disordered setting behave as those of passive particles in an effective potential $U(\mathbf{r})$. The statistics of $U(\mathbf{r})$ are those of a Gaussian surface [56] - a self-affine fractal with deep wells. This effective potential captures the component of the non-equilibrium current $\mathbf{j}$ which accounts for both the clustering and the long-range correlations observed in our numerics. (See Fig.1(b) and Supplementary Movies, which show how MIPS is destabilized by the introduction of the random potential.)

Persistent currents. The random force density $\mathbf{f}(\mathbf{r})$ is a nonconservative vector field due to the divergencefree part $\boldsymbol{\xi}(\mathbf{r})$. While this term does not influence the density field, it induces currents in the system. To quantify them, we consider a closed contour $\mathcal{C}$. Taking the curl of the total current and averaging over noise, one finds $\langle\nabla \times \mathbf{j}\rangle=\nabla \times \boldsymbol{\xi}$. Integrating this relation over a domain enclosed by $\mathcal{C}$, we obtain, using Stokes theorem, that the circulation of $\langle\mathbf{j}\rangle$ is entirely controlled by $\boldsymbol{\xi}: J(\mathcal{C}) \equiv \oint_{\mathcal{C}} \mathrm{d} \mathbf{l} \cdot \mathbf{j}(\mathbf{r})=\oint_{\mathcal{C}} \mathrm{d} \mathbf{l} \cdot \boldsymbol{\xi}(\mathbf{r}) . J(\mathcal{C})$ is thus a sum of uncorrelated random numbers and we predict its variance to scale as the perimeter of the contour $\mathcal{C}$, with a 
slope proportional to the disorder strength $\sigma$. This is confirmed by our numerics in Fig. 3(b). Furthermore, the steady-state current induced by a realization of the random potential is shown in Fig. 3)(a). It should be constrasted with the equilibrium case in which currents vanish in the steady-state.

Strong-disorder regime and self-consistency of the linear theory. The linear theory used in the previous section is valid as long as density fluctuations are small compared to the mean density. To detect a possible departure from this scenario, we measure the density fluctuations across a length $\ell$ through $\overline{\left\langle\delta \rho^{2}(\ell)\right\rangle}=2[g(a)-g(\ell)]$, with $a$ a short-distance cutoff. The fluctuations have to remain small compared to the natural scales of the density field: $\overline{\left\langle\delta \rho^{2}(\ell)\right\rangle} \ll \rho_{b}^{2}$ with $\rho_{b} \equiv \min \left(\rho_{0}, \rho_{M}-\rho_{0}\right)$. Here $\rho_{0}$ and $\rho_{M}$ are the average and maximal particle densities. Using Eq. (6), we find for large $\ell$

$$
\frac{\overline{\left\langle\delta \rho^{2}(\ell)\right\rangle}}{\rho_{b}^{2}}= \begin{cases}\frac{\sigma^{2} \ln (\ell / a)}{\pi u^{2} \rho_{b}^{2}} & \text { for } d=2 \\ \frac{\sigma^{2} a^{2-d}}{(d-2) S_{d} u^{2} \rho_{b}^{2}} & \text { for } d>2 .\end{cases}
$$

For $d>2$, the linear theory thus holds if $\sigma \ll$ $u \rho_{b} \sqrt{(d-2) S_{d} a^{d-2}}$, namely, whenever the disorder is weak enough. For strong enough disorder, the breakdown of the linear approximation indicates the possibility of a different behavior for $S(q)$. For $d=2$, the criterion is valid only for length scales satisfying $\ell \ll \ell^{*}$ with $\ell^{*} \equiv a \exp \left(\pi u^{2} \rho_{b}^{2} / \sigma^{2}\right)$. Note that this length scale is exponential in the square of the ratio between the effective temperature and the disorder strength, since $\sigma / u \propto \beta_{\text {eff }} \chi$. This can be estimated using Fig. 2] leading to very large length scales, well beyond the reach of our numerics. We suggest in the SI an alternative numerical approach to study the strong-disorder regime in $d=2$ using passive particles in a Gaussian surface. The resulting correlation function shows clear deviations from the logarithmic behavior on large length scales.

Lower critical dimension. Our linear theory offers an avenue to test the stability of phase separation against weak disorder. To do so, we note that the HelmholtzHodge decomposition implies that the dynamics of $\phi(\mathbf{r})$ are similar to an equilibrium dynamics in a correlated random potential $U(\mathbf{r})$. This allows employing an ImryMa argument 22, 23 in order to obtain the lower critical dimension $d_{c}$ below which phase separation is suppressed at large scales. To do so, we consider a domain of linear size $\ell$. The surface energy of the domain is given by $\gamma \ell^{d-1}$ with $\gamma$ the surface tension [57]. On the other hand, the contribution of the disorder to the energy of the domain is, to leading order, $E(\ell)=\int_{\ell^{d}} \mathrm{~d}^{d} \mathbf{r}^{\prime} \rho_{0} U\left(\mathbf{r}^{\prime}\right)$. The typical energy of a domain of size $\ell$ is thus given by $\sqrt{\overline{E(\ell)^{2}}}=\sigma \rho_{0} \ell^{(d+2) / 2}$. Comparing the two energy scales shows the lower critical dimension to be $d_{c}=4$. In lower dimensions the contribution of the surface energy is neg-

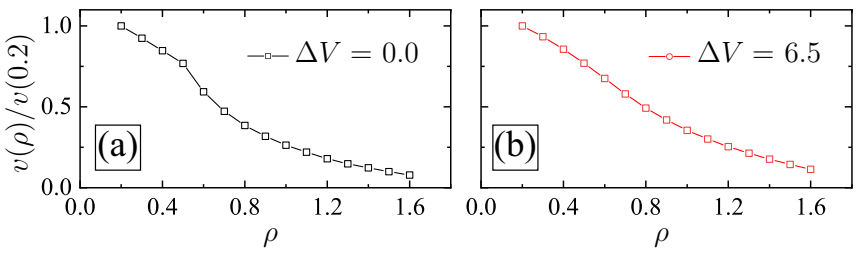

FIG. 4. Measurement of the effective self-propulsion speed $v(\rho)$ in our simulations without (a) and with (b) disorder for $v=13$ and $\alpha=1$. Both systems exhibit a similar decay which, in the absence of disorder, would lead to MIPS. Note that the kink observed for $v(\rho)$ in the left panel stems from the occurrence of phase separation.

ligible on large enough length scales and a system of size $L$ does not phase separate if $L \gg \ell_{I M} \simeq\left[\gamma /\left(\sigma \rho_{0}\right)\right]^{\frac{2}{4-d}}$, which we term the Imry-Ma length scale. Numerically, we indeed confirm that the coarsening to a single macroscopic domain is only observed for small system sizes. Correspondingly, a transition from linearly decaying to logarithmically decaying pair-correlation functions with increasing $L$ is reported in SI [48].

Note that the Imry-Ma argument rules out the existence of a macroscopic ordered/dense phase. Alternatively, the absence of MIPS could stem from the suppression of the feedback loop between a slowdown of particles at high density and their tendency to accumulate where they move slower. Reformulated as a mean-field theory, this feedback loop translates into an instability criteria for a homogeneous system of density $\rho$ whenever $\rho v^{\prime}(\rho)<-v(\rho)$ [29, 38], where $v(\rho)$ is an effective propulsion speed in a system of density $\rho$. We report in Fig. 4 the measurement of $v(\rho)$ for our system, defined as the mean hoping rate of particles along their orientation, with and without the random potential. Both systems show a similar decay which, at mean-field level, would predict the occurrence of MIPS. It is thus the non-trivial correlations induced by disorder that make MIPS disappear at large scales, despite an underlying instability at mean-field level. The disorder-induced disappearance of MIPS thus has a very different origin than its arrest by diffusiophoretic [58, 59] or hydrodynamic [60] interactions that directly prevent a kinetic hindrance at the microscopic scale.

Conclusion. In this Letter we have shown how random quenched potentials and torques lead to a non-trivial phase in scalar active matter with anomalous correlations that prevent phase separation. Interestingly, while the transition to collective motion is more robust to disorder than the corresponding ferromagnetic transition in equilibrium [16, 17], the converse holds for scalar phase separation: the lower critical dimension is larger in the active case $\left(d_{c}=4\right)$ than in the passive one $\left(d_{c}=2\right)$. We also note a strong difference between the one-dimensional active case, in which disorder promotes clustering [10], and the two-dimensional one, in which MIPS is destroyed by 
disorder at large scale. We expect our results, presented here for RTPs, to hold for generic scalar active systems, including active Brownian and active Ornstein Uhlenbeck particles. Experimentally, we expect our results to be relevant for a large class of systems. Long-ranged correlations, forces on obstacles, and current circulations could be tested using self-propelled colloids [61, 62] and shaken grains 63] on irregular surfaces - at least in their dilute, disordered phase - as well as swimming bacteria in disordered media [12, 13]. The suppression of phase separation and MIPS-related physics could be studied in experiments using self-propelled colloids [31, 64] or bacteria [65, [66]. It would also be of interest to see whether the bubbly phase, uncovered recently in [41, 67], exhibits different behavior under disorder. Finally, we note that random potentials lead to ratchets in many nonequilibrium systems, whether classical or quantum, far beyond the realm of active matter. Since these currents are the building blocks of our field-theoretical treatment, we expect our results to play a role in many nonequilibrium systems experiencing random potentials.

Acknowledgments: SR, YK \& MK were supported by an NSF5-BSF grant (DMR-170828008). SR \& YK were also supported by the Israel Science Foundation and the National Research Foundation of Korea (2019R1A6A3A03033761). SR, JT \& YK acknowledge support from a joint CNRS-MOST grant. The authors benefited from participation in the 2020 KITP program on Active Matter supported by the Grant NSF PHY1748958.

[1] O. Chepizhko and F. Peruani, Physical Review Letters 111, 160604 (2013).

[2] C. Reichhardt and C. J. Olson Reichhardt, Physical Review E 90, 012701 (2014).

[3] C. Bechinger, R. Di Leonardo, H. Löwen, C. Reichhardt, G. Volpe, and G. Volpe, Reviews of Modern Physics 88, 045006 (2016)

[4] E. Pinçe, S. K. P. Velu, A. Callegari, P. Elahi, S. Gigan, G. Volpe, and G. Volpe, Nature Communications 7, 10907 (2016).

[5] A. Morin, D. Lopes Cardozo, V. Chikkadi, and D. Bartolo, Physical Review E 96, 042611 (2017)

[6] C. Sándor, A. Libál, C. Reichhardt, and C. J. Olson Reichhardt, Physical Review E 95, 032606 (2017).

[7] F. Peruani and I. S. Aranson, Physical Review Letters 120, 238101 (2018).

[8] C. Reichhardt and C. J. O. Reichhardt, Physical Review E 97, 052613 (2018)

[9] R. L. Stoop and P. Pierno, Communications Physics 1, 68 (2018).

[10] Y. Ben Dor, E. Woillez, Y. Kafri, M. Kardar, and A. P. Solon, Physical Review E 100, 052610 (2019)

[11] P. L. Doussal, S. N. Majumdar, and G. Schehr, arXiv preprint arXiv:2003.08155 (2020).

[12] T. Bhattacharjee and S. S. Datta,
Soft Matter 15, 9920 (2019)

[13] T. Bhattacharjee and S. S. Datta, Nature Communications 10, 2075 (2019).

[14] E. Woillez, Y. Kafri, and N. S. Gov, Physical Review Letters 124, 118002 (2020).

[15] R. Das, M. Kumar, and S. Mishra, Physical Review E 98, 060602 (2018).

[16] J. Toner, N. Guttenberg, and Y. Tu, Physical Review E 98, 062604 (2018)

[17] J. Toner, N. Guttenberg, and Y. Tu, Physical Review Letters 121, 248002 (2018).

[18] A. Maitra, Physical Review E 101, 012605 (2020).

[19] O. Chepizhko, E. G. Altmann, and F. Peruani, Physical Review Letters 110, 238101 (2013).

[20] A. Morin, N. Desreumaux, J.-B. Caussin, and D. Bartolo, Nature Physics 13, 63 (2017)

[21] A. Chardac, S. Shankar, M. C. Marchetti, and D. Bartolo, arXiv:2002.12893 [cond-mat] (2020), arXiv: 2002.12893.

[22] Y. Imry and S.-k. Ma, Physical Review Letters 35, 1399 (1975)

[23] A. Aharony, Y. Imry, and S.-k. Ma, Physical Review Letters 37, 1364 (1976)

[24] D. S. Fisher, J. Fröhlich, and T. Spencer, Journal of Statistical Physics 34, 863 (1984)

[25] D. P. Belanger, A. R. King, V. Jaccarino, and J. L. Cardy, Physical Review B 28, 2522 (1983).

[26] J. Z. Imbrie, Physical Review Letters 53, 1747 (1984).

[27] J. Bricmont and A. Kupiainen, Physical Review Letters 59, 1829 (1987)

[28] U. Glaus, Physical Review B 34, 3203 (1986)

[29] J. Tailleur and M. Cates, Physical review letters 100, 218103 (2008).

[30] Y. Fily and M. C. Marchetti, Physical Review Letters 108, 235702 (2012)

[31] I. Buttinoni, J. Bialké, F. Kümmel, H. Löwen, C. Bechinger, and T. Speck, Physical Review Letters 110, 238301 (2013).

[32] M. E. Cates and J. Tailleur, EPL (Europhysics Letters) 101, 20010 (2013)

[33] J. Stenhammar, A. Tiribocchi, R. J. Allen, D. Marenduzzo, and M. E. Cates, Physical Review Letters 111, 145702 (2013).

[34] G. S. Redner, M. F. Hagan, and A. Baskaran, Physical Review Letters 110, 055701 (2013).

[35] A. P. Solon, M. E. Cates, and J. Tailleur, The European Physical Journal Special Topics 224, 1231 (2015)

[36] G. S. Redner, C. G. Wagner, A. Baskaran, and M. F. Hagan, Physical Review Letters 117, 148002 (2016).

[37] S. Paliwal, J. Rodenburg, R. van Roij, and M. Dijkstra, New Journal of Physics 20, 015003 (2018)

[38] M. E. Cates and J. Tailleur, Annual Review of Condensed Matter Physics 6, 219 (2015).

[39] A. P. Solon, J. Stenhammar, M. E. Cates, Y. Kafri, and J. Tailleur, Physical Review E 97, 020602 (2018)

[40] A. P. Solon, J. Stenhammar, M. E. Cates, Y. Kafri, and J. Tailleur, New Journal of Physics 20, 075001 (2018)

[41] E. Tjhung, C. Nardini, and M. E. Cates, Physical Review X 8, 031080 (2018)

[42] A. G. Thompson, J. Tailleur, M. E. Cates, and R. A. Blythe, Journal of Statistical Mechanics: Theory and Experiment 2011, P02

[43] M. Kourbane-Houssene, C. Erignoux, T. Bodineau, and J. Tailleur, Physical Review Letters 120, 268003 (2018) 
[44] S. Whitelam, K. Klymko, and D. Mandal, The Journal of Chemical Physics 148, 154902 (2018)

[45] J. Palacci, S. Sacanna, A. P. Steinberg, D. J. Pine, and P. M. Chaikin, Science 339, 936 (2013).

[46] D. Geyer, D. Martin, J. Tailleur, and D. Bartolo, Physical Review X 9, 031043 (2019)

[47] M. Kardar, Statistical Physics of Fields (Cambridge University Press, 2007).

[48] See Supplemental Material [url], which also includes descriptions of the movies and references to [68, 69].

[49] R. Soto and R. Golestanian, Physical Review E 89, 012706 (2014).

[50] N. Sepúlveda and R. Soto, Physical Review E 94, 022603 (2016).

[51] Y. Baek, A. P. Solon, X. Xu, N. Nikola, and Y. Kafri, Physical Review Letters 120, 058002 (2018).

[52] O. Granek, Y. Baek, Y. Kafri, and A. P. Solon, Journal of Statistical Mechanics: Theory and Experiment

[53] D. S. Fisher, Physical Review A 30, 960 (1984).

[54] J.-P. Bouchaud, A. Comtet, A. Georges, and P. Le Doussal, Annals of Physics 201, 285 (1990).

[55] With interactions, one finds $\chi / P^{\prime}\left(\rho_{0}\right)=\sigma / u$, with $P$ the pressure of the active fluid [52, 70].

[56] $U(\mathbf{r})$ can be constructed from $\mathbf{f}(\mathbf{r})$ by solving $\nabla^{2} U(\mathbf{r})=$ $\nabla \cdot \mathbf{f}(\mathbf{r})$.

[57] Unlike equilibrium systems, $\gamma$, when defined through momentum fluxes, can be negative for scalar active matter with pairwise forces, leading to a bubbly liquid phas [41, 67]. We expect our result to hold provided that the cost of the domain wall in the steady-state distribution increases as $\ell^{d-1}$ which is needed for overall phase separation between dilute-gas and bubbly-liquid phases.
[58] O. Pohl and H. Stark, Physical review letters 112, 238303 (2014).

[59] A. Zöttl and H. Stark, Physical review letters 112, 118101 (2014).

[60] R. Matas-Navarro, R. Golestanian, T. B. Liverpool, and S. M. Fielding, Physical Review E 90, 032304 (2014).

[61] J. R. Howse, R. A. Jones, A. J. Ryan, T. Gough, R. Vafabakhsh, and R. Golestanian, Physical review letters 99, 048102 (2007).

[62] A. Bricard, J.-B. Caussin, N. Desreumaux, O. Dauchot, and D. Bartolo, Nature 503, 95 (2013).

[63] J. Deseigne, O. Dauchot, and H. Chaté, Physical review letters 105, 098001 (2010).

[64] M. N. van der Linden, L. C. Alexander, D. G. Aarts, and O. Dauchot, Physical review letters 123, 098001 (2019).

[65] G. Liu, A. Patch, F. Bahar, D. Yllanes, R. D. Welch, M. C. Marchetti, S. Thutupalli, and J. W. Shaevitz, 2020, Dobjesida(2026) w letters 122, 248102 (2019).

[66] A. Curatolo, N. Zhou, Y. Zhao, C. Liu, A. Daerr, J. Tailleur, and J. Huang, Nature Physics 16, 1152 (2020).

[67] X.-q. Shi, G. Fausti, H. Chate, C. Nardini, and A. Solon, Physical Review Letters, 6 (2020).

[68] A. P. Solon, Y. Fily, A. Baskaran, M. E. Cates, Y. Kafri, M. Kardar, and J. Tailleur, Nature Physics 11, 673 (2015).

[69] Y. Fily, Y. Kafri, A. P. Solon, J. Tailleur, and A. Turner, Journal of Physics A: Mathematical and Theoretical 51, 044003 (2017).

[70] A. P. Solon, J. Stenhammar, R. Wittkowski, M. Kardar, Y. Kafri, M. E. Cates, and J. Tailleur, Physical Review Letters 114, 198301 (2015). 


\title{
Supplemental Information of Manuscript "Disorder-Induced Long-Ranged Correlations in Scalar Active Matter"
}

\author{
Sunghan Ro, ${ }^{1}$ Yariv Kafri, ${ }^{1}$ Mehran Kardar, ${ }^{2}$ and Julien Tailleur ${ }^{3}$ \\ ${ }^{1}$ Department of Physics, Technion-Israel Institute of Technology, Haifa, 3200003, Israel. \\ ${ }^{2}$ Department of Physics, Massachusetts Institute of Technology, Cambridge, Massachusetts 02139, USA. \\ ${ }^{3}$ Université de Paris, laboratoire Matière et Systèmes Complexes (MSC), UMR 7057 CNRS, 75205 Paris, France.
}

\section{CONTENTS}

I. Phenomenological model

A. Analytical computation of the structure factor

B. Long-ranged correlations in dilute, disordered phases

II. Field-theoretic treatment

III. Strong-disorder regime

IV. Imry-Ma length scale

V. The Influence of Torques

VI. Description of Supplementary Movies

References

\section{PHENOMENOLOGICAL MODEL}

\section{A. Analytical computation of the structure factor}

Here we evaluate the structure factor based on the random dipole picture of the phenomenological model. The density $\phi(\mathbf{r})$ in the presence of the random dipole field $\mathbf{P}(\mathbf{r})$ reads

$$
\langle\phi(\mathbf{r})\rangle=\beta_{\mathrm{eff}} \int \mathrm{d} \mathbf{r}^{\prime} \mathbf{P}\left(\mathbf{r}^{\prime}\right) \cdot \nabla_{\mathbf{r}} G\left(\mathbf{r}-\mathbf{r}^{\prime}\right),
$$

where $G\left(\mathbf{r}-\mathbf{r}^{\prime}\right)$ is the Green function of the Poisson equation. Note that if we have a delta-distributed dipole $\mathbf{P}(\mathbf{r})=\mathbf{p} \delta^{d}\left(\mathbf{r}-\mathbf{r}_{0}\right)$ in a $d$-dimensional free space, Eq. (S1) reads

$$
\langle\phi(\mathbf{r})\rangle=\frac{\beta_{\mathrm{eff}}}{S_{d}} \frac{\left(\mathbf{r}-\mathbf{r}_{0}\right) \cdot \mathbf{p}}{\left|\mathbf{r}-\mathbf{r}_{0}\right|^{d}}
$$

which recovers Eq. (1) of the main text. In what follows we use the Fourier convention:

$$
f(\mathbf{q})=\frac{1}{\sqrt{V}} \int \mathrm{d}^{d} \mathbf{r} e^{-i \mathbf{q} \cdot \mathbf{r}} f(\mathbf{r}) \quad \text { and } \quad f(\mathbf{r})=\frac{1}{\sqrt{V}} \sum_{\mathbf{q}} e^{i \mathbf{q} \cdot \mathbf{r}} f(\mathbf{q}),
$$

with $V$ denoting the volume of the system. In the Fourier representation, the convolution in Eq. (S1) is written as a product

$$
\langle\phi(\mathbf{q})\rangle=\beta_{\mathrm{eff}} V^{1 / 2} i \mathbf{q} \cdot \mathbf{P}(\mathbf{q}) G(\mathbf{q}) .
$$

Here $G(\mathbf{q})=-V^{-1 / 2} q^{-2}$ with $q=|\mathbf{q}|$, and the first two moments of $\mathbf{P}(\mathbf{q})$ reads

$$
\begin{aligned}
\overline{P_{i}(\mathbf{q})} & =0, \\
\overline{P_{i}(\mathbf{q}) P_{j}\left(\mathbf{q}^{\prime}\right)} & =\chi^{2} \delta_{i j} \delta_{\mathbf{q},-\mathbf{q}^{\prime}} .
\end{aligned}
$$



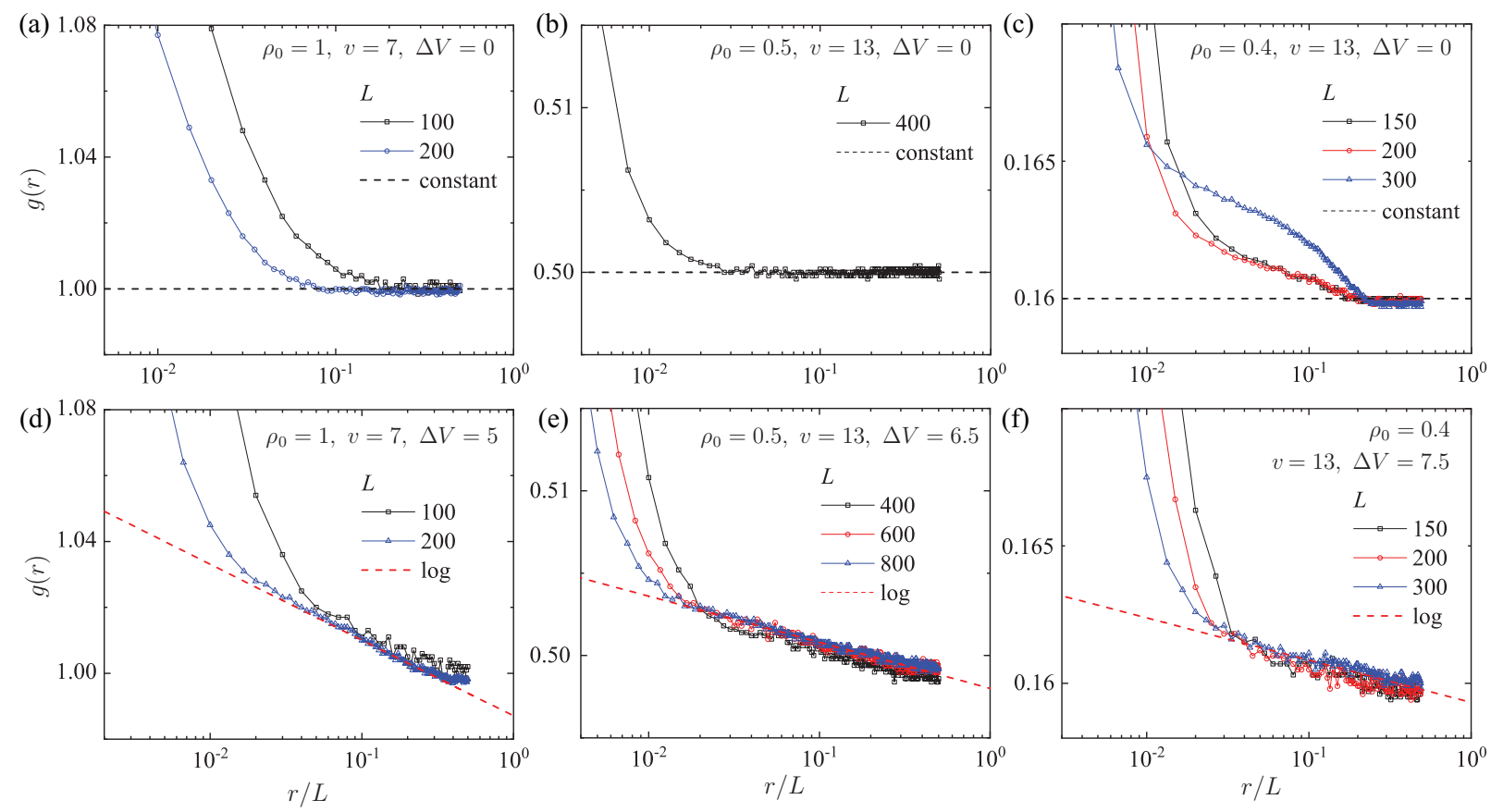

FIG. S1. Density two-point correlation functions $g(r)$ obtained from weekly propelling or dilute active matter. Upper panels: $g(r)$ obtained without disorder when (a) $v$ is small and (b) $\rho_{0}$ is small. Lower panels: $g(r)$ obtained with disorder when (c) $v$ is small and (d) $\rho_{0}$ is small. The panel (e) shows the correlation obtained from dilute active matter with the aligning interaction. Parameters: $\alpha=1, n_{M}=2, \tau=3.0, \theta_{M}=0.1$.

Using these relations, the structure factor is calculated in the dilute regime where $\overline{\langle\phi(\mathbf{q}) \phi(-\mathbf{q})\rangle}=\overline{\langle\phi(\mathbf{q})\rangle\langle\phi(-\mathbf{q})\rangle}$ as

$$
\begin{aligned}
\overline{\langle\phi(\mathbf{q}) \phi(-\mathbf{q})\rangle} & =\frac{\beta_{\text {eff }}^{2}}{q^{4}} \sum_{i, j=1}^{d} q_{i} q_{j} \overline{P_{i}(\mathbf{q}) P_{j}(-\mathbf{q})} \\
& =\frac{\beta_{\text {eff }}^{2} \chi^{2}}{q^{2}}
\end{aligned}
$$

which is Eq. (2) of the main text.

\section{B. Long-ranged correlations in dilute, disordered phases}

In Fig. S1, we show that the scale-invariant correlations are also observed in dilute systems. We consider three cases. First, we consider the model defined in the main text in the small $v$ or small $\rho_{0}$ regimes, where MIPS is not observed. In the absence of disorder, Fig. $\mathbf{S 1}(\mathrm{a})$ and (b) show density-density correlations $g(r)$ with short-ranged correlations. In the presence of disorder, Figs. S1(c) and (d) show the long-ranged logarithmic correlations predicted by Eq. (S3).

The third case we considered is the dilute, disordered phase of an active polar system. To do so, we introduce aligning interactions in the active lattice gas described in the main text. Specifically, given a particle $a$ at site $\vec{i}$ with orientation $\hat{e}_{\theta}^{a}$, we evaluate the local polarization of neighboring particles as $\mathbf{m}_{a ; \vec{i}}=\sum_{b \neq a} \hat{e}_{\theta}^{b}$, where $b$ runs over the particles at the site $\vec{i}$ and its nearest neighbors. In addition to hopping and uniform tumbles, the particles may now undergo a change of direction resulting from the aligning interactions. The corresponding rate $Y_{a}$ is given by

$$
Y_{a}=\tau n_{a}\left(1-\hat{m}_{a ; \vec{i}} \cdot\left\{\hat{e}_{\theta}\right\}_{a}\right)\left(1-\delta_{\mathbf{m}_{a ; \vec{i}}, 0}\right),
$$

where $\tau$ is the aligning interaction strength, $n_{a}$ is the number of neighboring particles used to calculate $\mathbf{m}_{a: \vec{i}}$, and $\hat{m}_{a ; \vec{i}}=\mathbf{m}_{a ; \vec{i}} /\left|\mathbf{m}_{a ; \vec{i}}\right|$. When such an aligning step occurs, the particle orientation change as $\theta \rightarrow \theta+\varepsilon \Delta \theta$ where $\Delta \theta \in\left[0, \theta_{M}\right]$ is drawn uniformly at random, and $\varepsilon= \pm 1$ is chosen to reduce the angle between $\hat{e}_{\theta}^{a}$ and $\mathbf{m}_{a ; \vec{i}}$. In Fig. S1 (c), we show that the system exhibits short-ranged correlations in its dilute, disordered phase. Note that 
aligning interactions make the correlation length larger than in the scalar system. In the presence of disorder, we again observe the predicted long-ranged correlations. This result confirms that, as expected, polar active systems exhibit the same structure factor as scalar active systems in their disordered phase. In experimental systems, the presence of aligning interactions will thus not be an obstacle to measuring long-ranged correlations as long as the systems are disordered.

\section{FIELD-THEORETIC TREATMENT}

Here we calculate the structure factor within the linear field-theory presented in the main text. Namely, the structure factor corresponding to

$$
\begin{aligned}
\frac{\partial}{\partial t} \phi(\mathbf{r}, t) & =-\nabla \cdot \mathbf{j}(\mathbf{r}, t), \\
\mathbf{j}(\mathbf{r}, t) & =-\nabla \mu[\phi]+\mathbf{f}(\mathbf{r})+\boldsymbol{\eta}(\mathbf{r}, t) .
\end{aligned}
$$

with

$$
\mu[\phi(\mathbf{r}, t)]=u \phi(\mathbf{r}, t)-K \nabla^{2} \phi(\mathbf{r}, t) .
$$

Here $\overline{f_{i}(\mathbf{r})}=0$ and $\overline{f_{i}(\mathbf{r}) f_{j}\left(\mathbf{r}^{\prime}\right)}=\sigma^{2} \delta_{i j} \delta^{d}\left(\mathbf{r}-\mathbf{r}^{\prime}\right)$. The Gaussian white noise $\boldsymbol{\eta}(\mathbf{r}, t)$ is characterized by zero mean and $\left\langle\eta_{i}(\mathbf{r}, t) \eta_{j}\left(\mathbf{r}^{\prime}, t^{\prime}\right)\right\rangle=2 D \delta_{i j} \delta^{d}\left(\mathbf{r}-\mathbf{r}^{\prime}\right) \delta\left(t-t^{\prime}\right)$.

Writing Eq. (S5) in Fourier space, we find

$$
\frac{\partial}{\partial t} \phi(\mathbf{q}, t)=-q^{2}\left(u+K q^{2}\right) \phi(\mathbf{q}, t)-i \mathbf{q} \cdot \mathbf{f}(\mathbf{q})-i \mathbf{q} \cdot \boldsymbol{\eta}(\mathbf{r}, t) .
$$

Solving this equation we obtain

$$
\phi(\mathbf{q}, t)=\phi(\mathbf{q}, 0) e^{-\kappa(\mathbf{q}) t}-\frac{i \mathbf{q} \cdot \mathbf{f}(\mathbf{q})}{\kappa(\mathbf{q})}\left(1-e^{-\kappa(\mathbf{q}) t}\right)-\int_{0}^{t} \mathrm{~d} t^{\prime} e^{-\kappa(\mathbf{q})\left(t-t^{\prime}\right)} i \mathbf{q} \cdot \boldsymbol{\eta}(\mathbf{r}, t) .
$$

Here $\kappa(\mathbf{q}) \equiv q^{2}\left(u+K q^{2}\right)$. Using this solution, we calculate the structure factor in the stationary state $S(\mathbf{q})=$ $\lim _{t \rightarrow \infty}\langle\overline{\langle\phi(\mathbf{q}, t) \phi(-\mathbf{q}, t)\rangle}$. This leads to

$$
\begin{aligned}
S(\mathbf{q}) & =\frac{q^{2} \sigma^{2}}{\kappa(\mathbf{q}) \kappa(-\mathbf{q})}+\frac{2 D q^{2}}{\kappa(\mathbf{q})+\kappa(-\mathbf{q})} \\
& =\frac{\sigma^{2}}{q^{2}\left(u+K q^{2}\right)^{2}}+\frac{D}{\left(u+K q^{2}\right)},
\end{aligned}
$$

where we used the fact that the second moments of the random variables in the Fourier representation read $\overline{f_{i}(\mathbf{q}) f_{j}\left(\mathbf{q}^{\prime}\right)}=\sigma^{2} \delta_{i j} \delta_{\mathbf{q},-\mathbf{q}^{\prime}}^{d}$ and $\left\langle\eta_{i}(\mathbf{q}, t) \eta_{j}\left(\mathbf{q}^{\prime}, t^{\prime}\right)\right\rangle=2 D \delta_{i j} \delta_{\mathbf{q},-\mathbf{q}^{\prime}}^{d} \delta\left(t-t^{\prime}\right)$.

\section{STRONG-DISORDER REGIME}

The strong-disorder regime proved beyond the numerical reach of our lattice-based model. Nevertheless, we can use the field-theoretical model to propose a heuristic approach to study the pair correlation function. Note that this approach will thus ignore any non-equilibrium non-linear contributions that the field theory does not capture. Since the effective potential exhibit deep wells, which are dominant for determining the particle distribution, we expect these non-linear contributions to play a less significant role and to leave the results unaltered at a qualitative level.

To proceed further, we generate a random effective potential $U(\mathbf{r})$ that satisfies the statistics of the Gaussian surface. Then, we introduce particles interacting with excluded volume interaction. Given the diverging depth of the potential well, temperature is not expected to play a large role and we consider the system at $T=0$. To do so, we simply fill up the system from the bottom of its deepest minima. This could be thought of as filling the Fermi-sea of a two-dimensional Fermionic system in a Gaussian surface potential. Finally, we measure the pair correlation function and average over disorder realizations.

In Fig. S2(a) and S2(b), we show configurations obtained from the process described above. Note that the particle distribution is superficially similar to the configurations obtained from the simulations of run-and-tumble particles presented in Fig. 1(b) of the main text. In Fig. S2 (c) and (d), we present the pair correlation functions, which we 
(a)
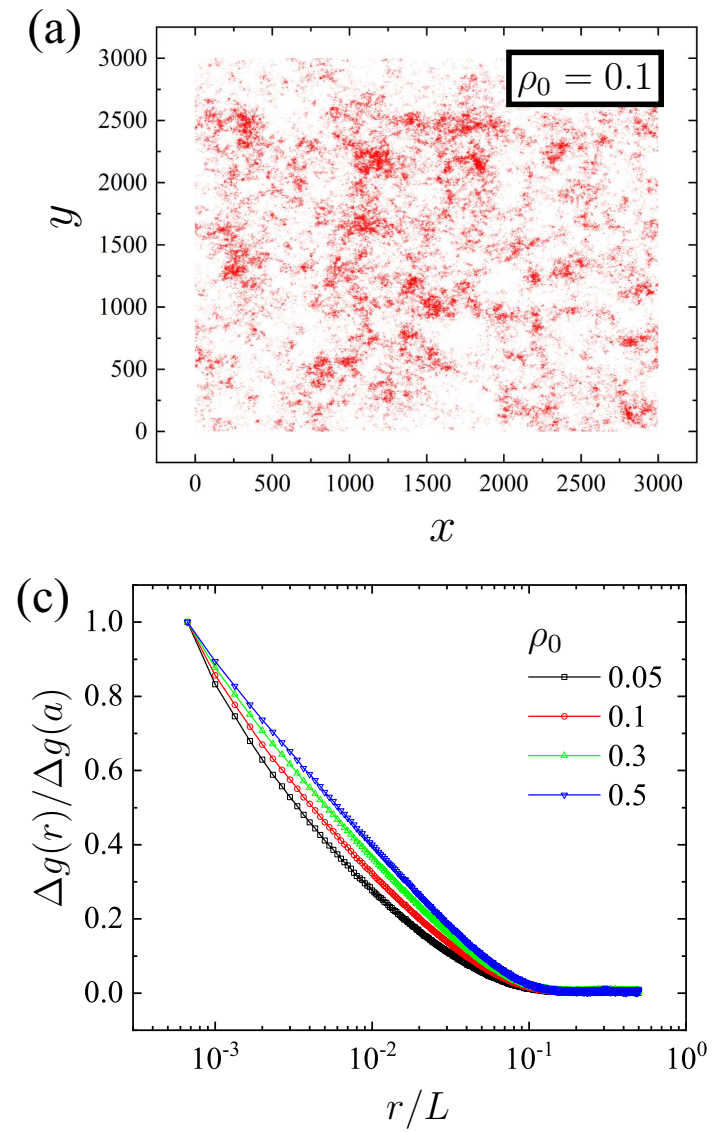

(b)

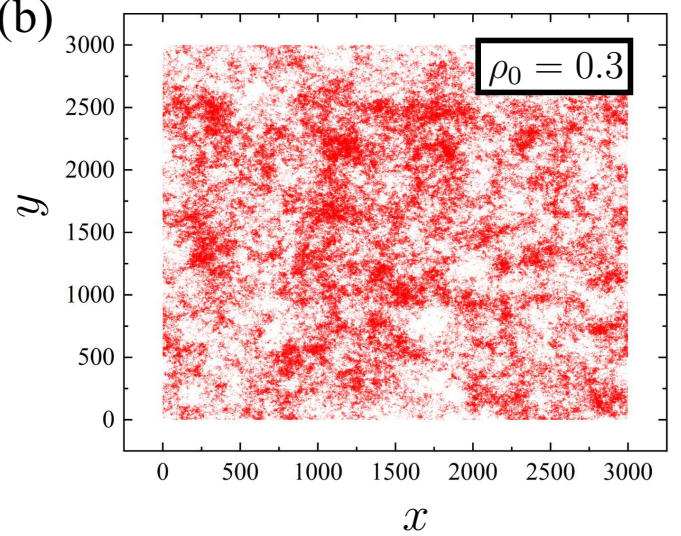

(d) 0.10

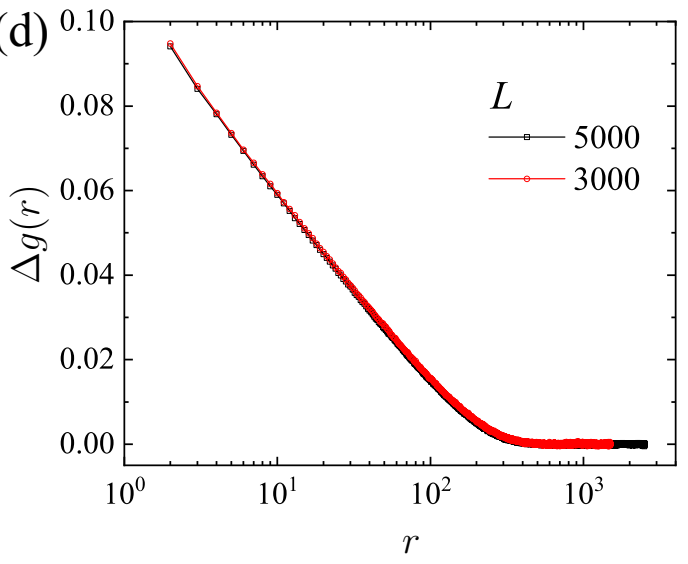

FIG. S2. Distribution of particles with an average density $\rho_{0}=0.1$ (a) and $\rho_{0}=0.3$ (b) on a Gaussian surface. The plateaux exhibited by the pair correlation functions, which we collapse at small and large lengths, in (c) show a clear departure from the logarithmic decay predicted in the weak disorder regime. Note that the crossover takes place at a length independent from system size, as shown in panel (d) by overlapping data for $L=3000$ and 5000 .

rescale as $\tilde{g}(r) \equiv \Delta g(r) / \Delta g(a)$ where $a$ is the lattice spacing $a$ and $\Delta g(r) \equiv g(r)-g(L / 2)$. As predicted, when $r / L$ is small, $\tilde{g}(r)$ shows a logarithmic decay as a function of $r / L$. For large $r / L$, however, the pair correlation function deviates from this logarithmic behavior, consistently with the prediction of a strong-disorder regime. In Fig. S2 (d), we show the crossover to be independent of system size, as predicted by our theory.

\section{IMRY-MA LENGTH SCALE}

In the following, we explore how the system transitions from phase-separated to homogeneous at large scales as the system size crosses the Imry-Ma length scale. To do so, we measure the pair-correlation function for different system sizes in the presence of disorder. We show an example of such a measurement in Fig. S3 (a) for a disorder strength $\Delta V=2.5$. As shown in this Figure, there are two distinct regimes which depend on the system size. The data are correspondingly marked in blue and red. The crossover length between the two behaviors provides an approximate measurement of $\ell_{\mathrm{IM}}$. Indeed, for $L \ll \ell_{\mathrm{IM}}: g(r)$ decays linearly with $r / L$ as expected for a phase-separated system (see blue curves and black dashed line). In contrast, for $L \gg \ell_{\mathrm{IM}}, g(r)$ decays logarithmically with $r / L$ as predicted in the main text and confirmed in Fig. S3(b) (see red curves and red dashed line). Sample configurations in each of the regimes are also shown as insets in Fig. S3(a).

\section{THE INFLUENCE OF TORQUES}

Here we consider the effect of torques acting on a dilute gas of ABPs or RTPs. In particular, we show that a localized torque influences the far field and current densities in the same manner as an asymmetric potential. The 

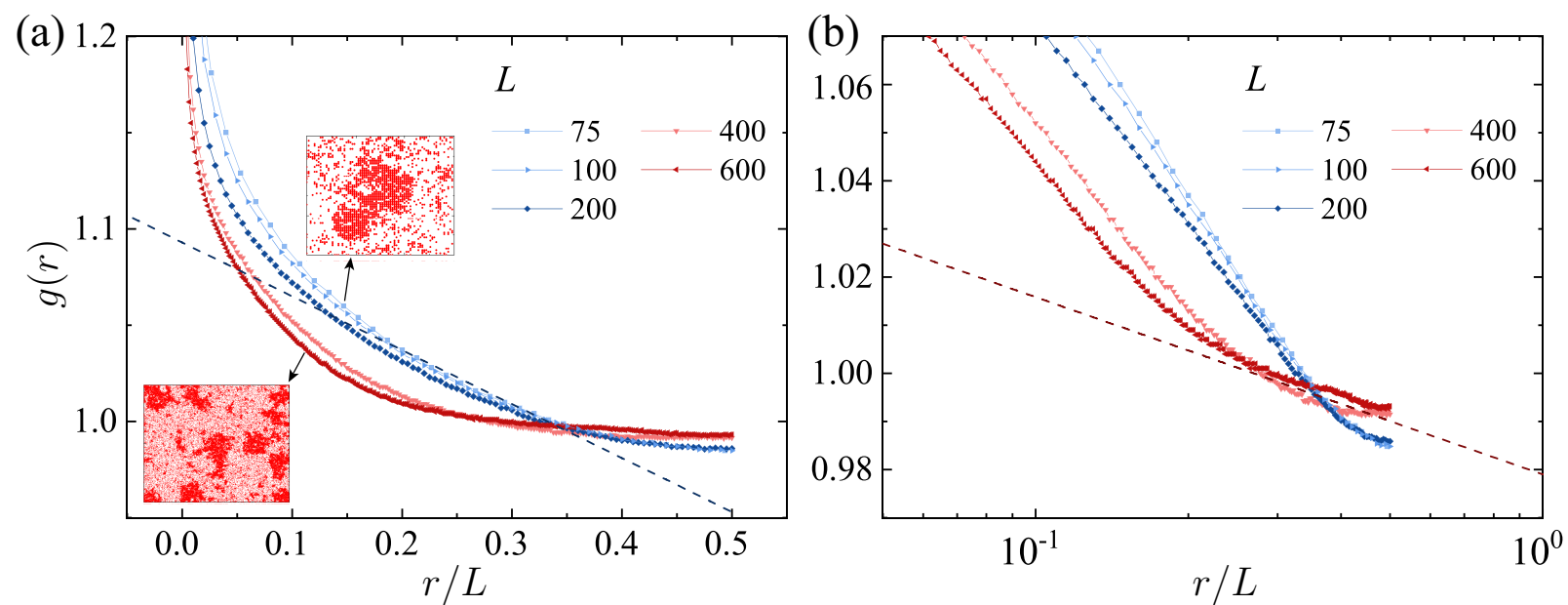

FIG. S3. The density pair correlation function measured for $\Delta V=2.5$ and $v / \alpha=10$ for different system sizes. The data in blue corresponds to small system sizes and in red to larger systems. Two different behaviors are observed consistent with the existence of an Imry-Ma length scale.

only difference is that the dipole strength is no longer simply related to the force exerted by the asymmetric potential on the active fluid. This result implies that the phenomenological model and the field theoretical treatments of the main text remain unchanged in the presence of torques. This statement is supported by numerical simulations in two dimensions which include interactions between the particles and confirm the expected decay of the correlation function.

To proceed, we follow Ref. [51] of the main text to derive the equation for the density field in the far-field of a localized torque. For completeness, we also account for a localized potential in the derivation. It should be clear to the reader that allowing only torques does not modify the results. In addition, for simplicity, the derivation is carried out in two-dimensions. The extension to higher dimensions is straightforward.

Consider the two-dimensional Fokker-Planck equation:

$$
\partial_{t} P=-\nabla \cdot\left[\left(v \mathbf{e}_{\theta}-\nabla V(\mathbf{r})-D_{t} \nabla\right) P\right]-\alpha P+\frac{\alpha}{2 \pi} \int \mathrm{d} \theta P-\partial_{\theta}[\Gamma(\mathbf{r}, \theta) P]+D_{r} \partial_{\theta}^{2} P .
$$

Here, $P(\mathbf{r}, \theta)$ is the probability density of finding the particle at position $\mathbf{r}$ with an orientation $\theta, v$ is the particle's speed, $\mathbf{e}_{\theta}$ is an unit vector in the $\theta$ direction, $V(\mathbf{r})$ is the potential, $D_{t(r)}$ is a translational (rotational) diffusion constant, and $\alpha$ is a tumbling rate. As in the main text, we set the mobilities to one. The localized torque exerted on the particle is accounted for through $\Gamma(\mathbf{r}, \theta)$. We assume that $V(\mathbf{r})$ and $\Gamma(\mathbf{r}, \theta)$ are non-zero in the same region of space.

Next, we introduce the marginal distributions for the probability density:

$$
\mathbf{m}^{(n)}(\mathbf{r}) \equiv \int \mathrm{d} \theta P(\mathbf{r}, \theta) \mathbf{e}_{\theta}^{(n)}
$$

with

$$
\mathbf{e}_{\theta}^{(n)} \equiv\left[\begin{array}{l}
\cos (n \theta) \\
\sin (n \theta)
\end{array}\right]
$$

Note that $\mathbf{m}^{(0)}(\mathbf{r}) \equiv[\rho(\mathbf{r}), 0]^{\mathrm{T}}$. We further define the marginal torque distributions

$$
\boldsymbol{\Gamma}^{(n)}(\mathbf{r}) \equiv \int \mathrm{d} \theta \Gamma(\mathbf{r}, \theta) P(\mathbf{r}, \theta) \mathbb{C e}_{\theta}^{(n)}
$$

with

$$
\mathbb{C} \equiv\left[\begin{array}{cc}
0 & -1 \\
1 & 0
\end{array}\right]
$$


Multiplying Eq. (S7) by $\mathbf{e}_{\theta}^{(n)}$ and integrating over $\theta$, one finds that the equations for the marginal distributions are given in the steady-state by

$$
\begin{aligned}
& 0=-\nabla \cdot\left(v \mathbf{m}^{(1)}-\rho \nabla V-D_{t} \nabla \rho\right) \\
& 0=-\left(\alpha+D_{r} n^{2}\right)\left(1-\mathbb{M}^{(n)}\right) \mathbf{m}^{(n)}-\frac{v}{2}\left(\mathbb{D} \mathbf{m}^{(n-1)}-\mathbb{D}^{\dagger} \mathbf{m}^{(n+1)}\right)+n \boldsymbol{\Gamma}^{(n)} .
\end{aligned}
$$

Here, the operators $\mathbb{M}^{(n)}, \mathbb{D}$, and $\mathbb{D}^{\dagger}$ are defined as

$$
\begin{aligned}
\mathbb{M}^{(n)} & \equiv \frac{1}{\alpha+D_{r} n^{2}}\left[\nabla \cdot(\nabla V)+D_{t} \nabla^{2}\right], \\
\mathbb{D} & \equiv\left[\begin{array}{cc}
\partial_{x} & -\partial_{y} \\
\partial_{y} & \partial_{x}
\end{array}\right], \quad \mathbb{D}^{\dagger} \equiv\left[\begin{array}{cc}
-\partial_{x} & -\partial_{y} \\
\partial_{y} & -\partial_{x}
\end{array}\right] .
\end{aligned}
$$

Using Eq. (S13), we can express $\mathbf{m}^{(1)}$ and $\mathbf{m}^{(2)}$ as

$$
\begin{aligned}
& \mathbf{m}^{(1)}=\mathbb{M}^{(1)} \mathbf{m}^{(1)}-\frac{l_{r}}{2}\left(\nabla \rho-\mathbb{D}^{\dagger} \mathbf{m}^{(2)}\right)+\frac{1}{\alpha+D_{r}} \boldsymbol{\Gamma}^{(1)} \\
& \mathbf{m}^{(2)}=\mathbb{M}^{(2)} \mathbf{m}^{(2)}-\frac{l_{r}}{2} \frac{\alpha+D_{r}}{\alpha+4 D_{r}}\left(\mathbb{D} \mathbf{m}^{(1)}-\mathbb{D}^{\dagger} \mathbf{m}^{(3)}\right)+\frac{2}{\alpha+4 D_{r}} \boldsymbol{\Gamma}^{(2)} .
\end{aligned}
$$

Here, $l_{r} \equiv v /\left(\alpha+D_{r}\right)$ is the run length. Expressing $-\nabla \cdot\left(v \mathbf{m}^{(1)}\right)$ in Eq. (S12) by combining Eqs. (S16) and (S17), one finds

$$
\begin{aligned}
-\nabla \cdot\left(v \mathbf{m}^{(1)}\right)= & -l_{r} \sum_{a, b} \partial_{a} \partial_{b}\left[\left(\partial_{a} V\right)\left(\mathbf{m}^{(1)} \cdot \mathbf{e}_{b}\right)\right]-D_{t} l_{r} \nabla^{2} \nabla \cdot \mathbf{m}^{(1)} \\
& +\frac{v l_{r}}{2} \nabla^{2} \rho-\frac{v l_{r}}{2} \nabla \cdot \mathbb{D}^{\dagger} \mathbf{m}^{(2)}-l_{r} \nabla \cdot \boldsymbol{\Gamma}^{(1)} \\
= & \frac{v l_{r}}{2} \nabla^{2} \rho-l_{r} \nabla \cdot \boldsymbol{\Gamma}^{(1)}+l_{r}^{2} \frac{\alpha+D_{r}}{\alpha+4 D_{r}} \nabla \cdot \mathbb{D}^{\dagger} \boldsymbol{\Gamma}^{(2)} \\
& -l_{r} \sum_{a, b} \partial_{a} \partial_{b}\left[\left(\partial_{a} V\right)\left(\mathbf{m}^{(1)} \cdot \mathbf{e}_{b}\right)\right]+\sum_{a, b, c} \partial_{a} \partial_{b} \partial_{c} \mathbb{H}_{a b c},
\end{aligned}
$$

with

$$
\begin{aligned}
\sum_{a, b, c} \partial_{a} \partial_{b} \partial_{c} \mathbb{H}_{a b c}= & -D_{t} l_{r} \nabla^{2} \nabla \cdot \mathbf{m}^{(1)}-\frac{l_{r}^{2}}{2} \frac{\alpha+D_{r}}{\alpha+4 D_{r}} \nabla \cdot \mathbb{D}^{\dagger} \sum_{a} \partial_{a}\left[\left(\partial_{a} V\right)+D_{t} \partial_{a}\right] \mathbf{m}^{(2)} \\
& +\frac{v l_{r}^{2}}{4} \frac{\alpha+D_{r}}{\alpha+4 D_{r}} \nabla \cdot\left[\nabla^{2} \mathbf{m}^{(1)}-\left(\mathbb{D}^{\dagger}\right)^{2} \mathbf{m}^{(3)}\right] .
\end{aligned}
$$

Inserting Eq. (S18) into Eq. (S12), the following two-dimensional Poisson equation is obtained

$$
\begin{aligned}
\nabla^{2} \rho= & -\beta_{\mathrm{eff}} \nabla \cdot\left(\rho \nabla V-l_{r} \boldsymbol{\Gamma}^{(1)}\right) \\
& +\beta_{\mathrm{eff}}\left[-l_{r}^{2} \frac{\alpha+D_{r}}{\alpha+4 D_{r}} \nabla \cdot \mathbb{D}^{\dagger} \boldsymbol{\Gamma}^{(2)}+l_{r} \sum_{a, b} \partial_{a} \partial_{b}\left[\left(\partial_{a} V\right)\left(\mathbf{m}^{(1)} \cdot \mathbf{e}_{b}\right)\right]-\sum_{a, b, c} \partial_{a} \partial_{b} \partial_{c} \mathbb{H}_{a b c}\right], \\
= & -\beta_{\mathrm{eff}} \nabla \cdot \hat{\mathbf{p}}+\beta_{\mathrm{eff}} \hat{\mathbb{W}} .
\end{aligned}
$$

Here, $\beta_{\text {eff }} \equiv\left(D_{t}+v l_{r} / 2\right)^{-1}, \hat{\mathbf{p}} \equiv \rho \nabla V-l_{r} \boldsymbol{\Gamma}^{(1)}$, and $\widehat{\mathbb{W}}$ is the terms in the square brackets of Eq. (S20). Using the Green's function of the Laplace operator $G\left(\mathbf{r}-\mathbf{r}^{\prime}\right)$, we write

$$
\rho(\mathbf{r})=\int \mathrm{d} \mathbf{r}^{\prime} \beta_{\text {eff }} G\left(\mathbf{r}-\mathbf{r}^{\prime}\right)\left[-\nabla \cdot \hat{\mathbf{p}}\left(\mathbf{r}^{\prime}\right)+\hat{\mathbb{W}}\left(\mathbf{r}^{\prime}\right)\right] .
$$

Since the potential $V(\mathbf{r})$ and the torque $\Gamma(\mathbf{r}, \theta)$ are localized quantities, we can perform multipole expansion of the Green's function in the far-field regime. The multipole order of a term increases with the number of spatial gradients applied on it, and the leading order term in Eq. (S22) has a dipole form corresponding to terms with one spatial derivative. Using the two-dimensional expression for the Green's function, $G(\mathbf{r})=\ln |\mathbf{r}| /(2 \pi)$, then gives,

$$
\rho(\mathbf{r})=\rho_{0}+\frac{\beta_{\mathrm{eff}}}{2 \pi} \frac{\mathbf{r} \cdot \mathbf{p}}{r^{2}}+\mathcal{O}\left(r^{-2}\right)
$$



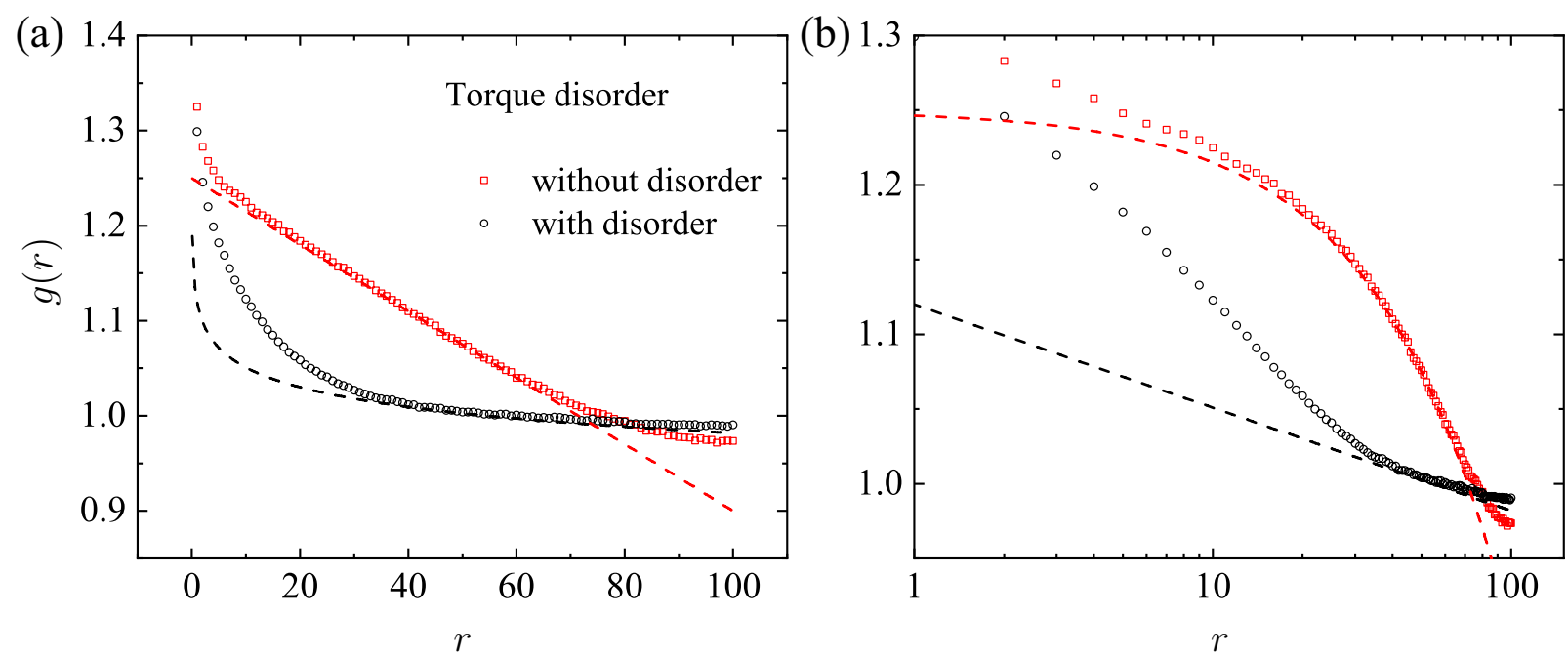

FIG. S4. The pair correlation functions shown in (a) a linear scale and (b) a log-linear scale. The simulation results obtained without (red symbols) and with (black symbols) disorder are shown with guidelines presenting linear (red dashed lines) and logarithmic (black dashed lines) decays. Parameters: $L=200, \Delta V=3, v=10, \alpha=1, \rho_{0}=1$, and $n_{M}=2$.

with

$$
\mathbf{p}=-\int \mathrm{d}^{2} \mathbf{r}^{\prime}\left[\rho \nabla^{\prime} V-l_{r} \boldsymbol{\Gamma}^{(1)}\right]
$$

In sum, we find that a localized torque, even in the absence of an asymmetric potential, leads to far field of the density and current which are identical to those of an asymmetric potential. The only difference is a renormalization of the dipole strength.

It is interesting to note that the amplitude of the dipole is directly related to the average rate of injection of momentum into the system due to both the external potential and the rectification of the random active motion by the torques. This is very similar to the role played by wall torques in destroying the equation of state for the pressure of active systems [1, 2].

We also remark that the results above can be derived straightforwardly for a potential $V(\mathbf{r}, \theta)$ which is a function of both $\mathbf{r}$ and $\theta$. In this case, none of the contributions to the dipole strength are directly related to the force exerted by the potential on the active particles.

To verify that interactions between particles do not change the results, we carried out numerical simulations in two dimensions. In order to emphasize that even random torques induce the same behavior as random potentials, our numerical simulations only consider the former and interactions between the particles. The dynamics are similar to the rules described in the main text. However, the disorder does not affect the translational hopping and instead only modifies the tumbling motion. The details of the dynamics are defined as follows. We consider run-and-tumble particles with continuous orientation $\hat{e}_{\theta}=(\cos \theta, \sin \theta)$ with $\theta \in[0,2 \pi)$. Due to activity, the particles hops from the initial position of a particle $\vec{i}$ to one of its neighbors $\vec{j}=\vec{i}+\hat{u}$ with rate $W_{\vec{i}, \vec{j}}=\max \left[v \hat{u} \cdot \hat{e}_{\theta}, 0\right]$ with $v$ controlling the propulsion speed. The steric repulsion between the particles modifies the hoping rates as $W_{\vec{i}, \vec{j}}^{\text {int }}=W_{\vec{i}, \vec{j}}\left(1-n_{\vec{j}} / n_{M}\right)$, where $n_{\vec{j}}$ is the number of particles at $\vec{j}$ and $n_{M}$ is the maximal particle number per site. The torque disorder biases the particle orientation toward an orientations $\theta_{\vec{i}}$, that are uniformly drawn in the range $[0,2 \pi)$ at random for each $\vec{i}$. In doing so, we mimic a torque exerted by a potential field of orientation $V_{\vec{i}}(\theta)$. To do so a particle at site $\vec{i}$ with orientation $\theta$ changes its orientation with the rate $\alpha Y_{\vec{i}}(\theta)$, where

$$
Y_{\vec{i}}(\theta)=\frac{1}{2 \pi} \int \mathrm{d} \theta^{\prime} e^{V_{\vec{i}}(\theta)-V_{\vec{i}}\left(\theta^{\prime}\right)} .
$$

The new orientation is chosen with probability density $\mathcal{P}(\theta)=e^{-V_{\vec{i}}(\theta)} /\left(Y_{\vec{i}}(\theta) e^{-V_{\vec{i}}(\theta)}\right)$. In the simulations, we use $V_{\vec{i}}(\theta)=-\Delta V \cos ^{2}\left(\theta-\theta_{\vec{i}}\right)$.

Figure 54 shows the results of our numerics. We chose a set of parameters which induce MIPS without disorder, and as a result, the pair correlation function obtained without disorder shown with red symbols in Fig. S4(a) presents linear decay, indicating phase separation. On the other hand, the black symbols obtained with torque disorder show 
logarithmic decay, as verified by the agreement between the black symbols and the black dashed line in Fig. S4(b). Thus, our simulation shows that MIPS break-down by the torque disorder, and the resulting disordered phase has the long-ranged density-density correlation similarly to the random potential considered in the main text.

\section{DESCRIPTION OF SUPPLEMENTARY MOVIES}

We provide movies capturing the time evolution of the particle density field obtained from simulations. In all the movies, we have $L=300, v=13, \alpha=1, n_{M}=2$, and $\rho_{0}=1$. A detailed descriptions of each movie is given as follows:

- Movie_1_v13_Vx.gif: These movies show the time evolution of the particle distribution starting from a homogeneous initial condition. The number $\mathrm{x}$ specifies the potential strength $\Delta V$.

- Movie_2_v13_V7.5_growing.gif: Here the strength of the disorder, $\Delta V$, is gradually increased from 0 to 7.5 . The initial state of the system is phase separated and one can observe how long-range order is lost following the introduction of disorder.

- Movie_2_v13_V7.5_vanishing.gif: In this movie, we gradually reduce the strength of the random potential $\Delta V$ from 7.5 to 0 . The initial configuration of the movie is the stationary configuration obtained with $\Delta V=7.5$. The movie shows how phase separation appears as the disorder is turned off.

[1] A. P. Solon, Y. Fily, A. Baskaran, M. E. Cates, Y. Kafri, M. Kardar, and J. Tailleur, Nature Physics 11, 673 (2015).

[2] Y. Fily, Y. Kafri, A. P. Solon, J. Tailleur, and A. Turner, Journal of Physics A: Mathematical and Theoretical 51, 044003 (2017). 